\title{
THE FORMAL MODULAR INVARIANT THEORY OF BINARY
}

\author{
QUANTICS* \\ BY \\ O. E. GLENN
}

The group, $G$, represented by the general binary linear transformation in which the coefficients are parameters representing residues of the prime number $p$, has the universal covariants $\dagger$

$$
L_{t}=x_{1}^{p t} x_{2}-x_{1} x_{2}^{p t},
$$

a fundamental system being given by $L=L_{1}, Q=L_{2} / L_{1}$. Assume that

$$
f_{m}=\left(a_{0}, a_{1}, \cdots, a_{m} \chi x_{1}, x_{2}\right)^{m}
$$

is a binary quantic of order $m$ whose coefficients are variables. We study the functions of the $a$ 's and $x$ 's which are invariantive under the operation of transforming $f_{m}$ by $G$. Sections $1,2,3$ deal with methods of constructing such functions, especial attention being given to the case $p=2$. In $\S 5$ fundamental systems of first degree concomitants of the quartic and quintic are derived. Section 4 is devoted to a proof that if the systems of concomitants modulo 2 of the forms of orders $1,2,3$ and the simultaneous systems obtained by combining forms of these three orders are all finite, then the system of $f_{m}$ is likewise finite. The hitherto undemonstrated theorem on the finiteness of the formal concomitants modulo 2 is thus reduced to a comparatively simple problem. In $\S 6$ there is proved a theorem on the reducibility of any covariant, modulo 2 , of the binary cubic in terms of a set of fourteen invariants and covariants.

\section{INVARIANT OPERATORS}

In addition to modular polars and transvectants previously discussed $\ddagger$ by the present writer we cite, in order to augment the number of construction

* Presented to the Society, February 26, 1916.

† Dickson, these Trans a ctions, vol. 12 (1911), p. 75; and Madison Colloquium Lectures, 1913, p. 33.

† O. E. Glenn, American Journal of Mathematics, vol. 37 (1915), p. 73; and Bulletin of the American Mathematical Society, vol. 21 (1915), p. 167. 
methods, the universal operators obtained by replacing, in $L$ and $Q$, the variables $x_{1}, x_{2}$ by the cogredient elements $\partial / \partial x_{2},-\partial / \partial x_{1}$. Call these operators respectively $L_{\delta}$ and $Q_{\delta}$;

$$
Q_{\delta}=\frac{\partial^{p(p-1)}}{\partial x_{1}^{p(p-1)}}+\frac{\partial^{p(p-1)}}{\partial x_{1}^{(p-1)(p-1)} \partial x_{2}^{p-1}}+\cdots .
$$

If $K_{M}$ is any formal covariant modulo $p$ of degree-order $(i, M)$ and if

$$
(p+1) r+p(p-1) s+w=M,
$$

then

$$
C_{r s w}=L_{\delta}^{r} Q_{\delta}^{s} K_{M}
$$

is a formal covariant of degree-order $(i, w)$. The number of concomitants yielded by this formula equals the number of distinct solutions in positive integers $(r, s, w)$ of the linear diophantine equation (1). The lists given below are for $p=2, K_{M} \equiv f_{m}$, each giving all forms $C_{r s w}$ for the corresponding $m$. Corresponding to each invariant $C_{r s 0}$ there exists an invariant operator $\sum(\partial / \partial a)$ obtained by constructing the Aronhold operator for the two forms $L^{r} Q^{s}, f_{m}$. These are also given in the particular cases shown.

$$
\begin{aligned}
& m=4 \\
& C_{020}=a_{1}+a_{2}+a_{3} ; \quad \frac{\partial}{\partial a_{0}}+\frac{\partial}{\partial a_{2}}+\frac{\partial}{\partial a_{4}}, \\
& C_{101}=a_{1} x_{1}+a_{3} x_{2}, \quad C_{012}=a_{1} x_{1}^{2}+a_{3} x_{2}^{2} . \\
& m=5 \\
& C_{110}=a_{1}+a_{2}+a_{3}+a_{4} ; \quad \frac{\partial}{\partial a_{1}}+\frac{\partial}{\partial a_{4}}, \\
& C_{021}=a_{2} x_{1}+a_{3} x_{2}, \quad C_{102}=a_{2} x_{1}^{2}+a_{3} x_{2}^{2}, \quad C_{013}=Q C_{021} . \\
& m=6 \\
& C_{200}=C_{030}=a_{3} ; \quad \frac{\partial}{\partial a_{0}}+\frac{\partial}{\partial a_{1}}+\frac{\partial}{\partial a_{3}}+\frac{\partial}{\partial a_{5}}+\frac{\partial}{\partial a_{6}} ; \quad \frac{\partial}{\partial a_{2}}+\frac{\partial}{\partial a_{4}}, \\
& C_{111}=\left(a_{1}+a_{3}\right) x_{1}+\left(a_{3}+a_{5}\right) x_{2}, \quad C_{022}=a_{3} Q, \quad C_{103}=a_{3} L, \\
& C_{014}=a_{1} x_{1}^{4}+a_{3} x_{1}^{2} x_{2}^{2}+a_{5} x_{2}^{4} \text {. } \\
& m=7 \\
& C_{120}=a_{1}+a_{2}+a_{3}+a_{4}+a_{5}+a_{6} ; \quad \sum_{i=1}^{6} \frac{\partial}{\partial a_{i}}, \\
& C_{201}=\left(a_{2}+a_{4}\right) x_{1}+\left(a_{3}+a_{5}\right) x_{2} \text {, } \\
& C_{031}=\left(a_{0}^{\prime \prime}+a_{1}+a_{3}+a_{5}+a_{6}\right) x_{1}+\left(a_{1}+a_{2}+a_{4}+a_{6}+a_{7}\right) x_{2} \text {, }
\end{aligned}
$$




$$
\begin{aligned}
& C_{112}=\left(a_{1}+a_{4}\right) x_{1}^{2}+\left(a_{3}+a_{6}\right) x_{2}^{2}, \\
& C_{023}=\left(a_{0}+a_{2}+a_{4}\right) x_{1}^{3}+\left(a_{1}+a_{3}+a_{5}\right) x_{1}^{2} x_{2}+\left(a_{2}+a_{4}+a_{6}\right) x_{1} x_{2}^{2} \\
& C_{104}=\left(a_{1}+a_{2}\right) x_{1}^{4}+\left(a_{5}+a_{6}\right) x_{2}^{4}, \\
& +\left(a_{3}+a_{5}+a_{7}\right) x_{2}^{3}, \\
& C_{015}=\left(a_{0}+a_{1}+a_{2}\right) x_{1}^{5}+\left(a_{1}+a_{2}+a_{3}\right) x_{1}^{4} x_{2}+\left(a_{4}+a_{5}+a_{6}\right) x_{1} x_{2}^{4} \\
& +\left(a_{5}+a_{6}+a_{7}\right) x_{2}^{5} \text {. }
\end{aligned}
$$

\section{Covariants Led BY ASSigned seminvariants}

If we add to the variables $a_{0}, a_{1}, \cdots ; x_{1}, x_{2}$ in any formal covariant $\phi\left(a_{0}, \cdots ; x_{1}, x_{2}\right)$ of $f_{m}$ the increments of these variables when $f_{m}$ is transformed by $x_{1}=x_{1}^{\prime}, x_{2}=t x_{1}^{\prime}+x_{2}^{\prime}$, and then expand $\phi\left(a_{0}+\delta a_{0}, \cdots\right)$ by Taylor's theorem, we find that any formal covariant modulo $p$ has an annihilator of the type*

$$
\Upsilon=O_{0}+O_{1} x_{1} \frac{\partial}{\partial x_{2}}+O_{2} x_{1}^{2} \frac{\partial^{2}}{\partial x_{2}^{2}}+\cdots+O_{j} x_{1}^{j} \frac{\partial^{j}}{\partial x_{2}^{j}}+\cdots .
$$

In this theory $t$ is any residue modulo $p$ and the expansion in question contains only the $p$ terms obtained by reducing all powers of $t$ below the $p$ th by Fermat's theorem. The operator $O_{j}(j=0,1, \cdots)$ is a partial differential operator in the derivatives with respect to the coefficients of $f_{m}$, non-homogeneous as to the derivatives the orders of which range from zero to infinity in each $O_{j}$.

If we apply $\Upsilon$ to a covariant and proceed as in the well-known proof of Roberts' theorem on the unique determination of an algebraical covariant from its seminvariant leader we find that the resulting relations in the covariant's coefficients and the operators $O_{j}(j=0,1, \cdots)$ are not recurrent in the formal theory, whereas they are recurrent in the algebraic theory. Thus a formal covariant is not uniquely determined from its leader. This is also evident from the fact that if $T$ is any such covariant of order $w \neq 0$ $(\bmod p)$, then

$$
\left(x_{1}^{p} \frac{\partial}{\partial x_{1}}+x_{2}^{p} \frac{\partial}{\partial x_{2}}\right) T
$$

is also a formal covariant having the same seminvariant leader.

Under definite conditions we can, however, derive a covariant $T$ of order $p-1$ of $f_{m}$ having a given seminvariant $S$ as leader. This is done by substituting for $a_{0}, a_{1}, a_{2}, \cdots$ in $S$ the derivatives

*Cf. Dickson, these Transactions, vol. 8 (1907), p. 209. An explicit $\Omega_{0}(\delta)$, analogous to $O_{0}$, is given in my paper in A merican Journal of Mathematics, loc. cit., p. 75. 


$$
f_{m}, \quad \frac{1}{m} \frac{\partial f_{m}}{\partial x_{2}}, \quad \frac{1}{m(m-1)} \frac{\partial^{2} f_{m}}{\partial x_{2}^{2}}, \cdots,
$$

regarding $x_{1}, x_{2}$ as integers modulo $p$ and rearranging the result as a polynomial in $x_{1}, x_{2}$ of order $p-1$. This is analogous to the process of Faà di Bruno for algebraic covariants. The same result is reached by expanding $S\left(a_{0}^{\prime}, a_{1}^{\prime}, \cdots\right)$, corresponding to the transformation of $f_{m}$ by

$$
x_{1}=x_{1}^{\prime}, \quad x_{2}=t x_{1}^{\prime}+x_{2}^{\prime},
$$

replacing $t$ by $x_{2} / x_{1}$ and multiplying by $x_{1}^{p-1}$. The result is

$$
T=S x_{1}^{p-1}+\frac{O_{0} S}{\underline{1}} x_{1}^{p-2} x_{2}+\cdots+\frac{O_{0}^{p-1} S}{\lfloor p-1} x_{2}^{p-1} .
$$

This is a formal covariant only provided it satisfies the differential congruence $\Upsilon T \equiv 0(\bmod p)$, and is symmetrical under the substitution

$$
s=\left(a_{0} a_{m}\right)\left(a_{1} a_{m-1}\right) \cdots\left(x_{1} x_{2}\right) .
$$

Imposing these conditions we deduce the conditions which $S$ must satisfy in order that it may lead the covariant of order $p-1$. These are, (a) that $S$ should be transformed into $O_{0}^{p-1} S / \mid \underline{p-1}$ by $s$, and (b) that the following congruences should be satisfied:

$$
\begin{aligned}
& O_{0}^{p} S \equiv 0 \text {, } \\
& \left(O_{0}^{p-1}+O_{1} O_{0}^{p-1}\right) S \equiv 0 \text {, } \\
& \left(O_{0}^{p-2}+O_{1} O_{0}^{p-2}+O_{2} O_{0}^{p-1}\right) S \equiv 0 \text { ， } \\
& \left(O_{0}^{2}+O_{1} O_{0}^{2}+O_{2} O_{0}^{3}+\cdots+O_{p-2} O_{0}^{p-1}\right) S \equiv 0 \text {, } \\
& \left(O_{0}+O_{1} O_{0}+O_{2} O_{0}^{2}+\cdots+O_{p-1} O_{0}^{p-1}\right) S \equiv 0 \text {. }
\end{aligned}
$$

The theory of $T$ is thus complicated. It is given here because nothing has been published hitherto on the determination of a covariant with a given leader, and also for the reason that it is of much practical use in constructing covariants with assigned properties in special cases. Let $p=2, m=3$. A seminvariant of $f_{3}$ is $S=a_{0} a_{2}+a_{1}^{2}$. Transformation of $f_{3}$ by $x_{1}=x_{1}^{\prime}$, $x_{2}=t x_{1}^{\prime}+x_{2}^{\prime}$ gives

$$
\begin{aligned}
S^{\prime} & \equiv\left[a_{0}+t\left(a_{1}+a_{2}+a_{3}\right)\right]\left(a_{2}+t a_{3}\right)+\left(a_{1}+t a_{3}\right)^{2} \\
& \equiv a_{0} a_{2}+a_{1}^{2}+\left(a_{0} a_{3}+a_{1} a_{2}+a_{1} a_{3}+a_{2}^{2}\right) t \quad(\bmod 2) .
\end{aligned}
$$

Rendering $S$ symmetrical with the coefficient of $t$ (condition $(a)$ ) by adding the invariant $a_{0} a_{3}+a_{1} a_{2}=\Delta$, (annihilated by $O_{0}$ ) we have the formal linear covariant*

*Cf. American Journal of Mathematics, loc. cit., p. 78. 


$$
C_{1}^{(2)}=(S+\Delta) x_{1}+\left(\Delta+a_{1} a_{3}+a_{2}^{2}\right) x_{2} .
$$

For $p=3, m=2, S=a_{0} a_{1}^{3}-a_{0}^{3} a_{1}$, the method leads easily to the quadratic covariant of $f_{2}$,

$$
C_{6}=S x_{1}^{2}+\left(a_{0} a_{2}^{3}-a_{0}^{3} a_{2}\right) x_{1} x_{2}+\left(a_{1} a_{2}^{3}-a_{1}^{3} a_{2}\right) x_{2}^{2} .
$$

3. Some covariants of $f_{m}$ modulo 2

For the modulus 2 the real points $\left(x_{1}, x_{2}\right)$, other than $(0,0)$, in the plane are $(1,1),(0,1),(1,0)$. The values of $f_{m}$ at these points are, respectively,

$$
a_{0}+a_{1}+\cdots+a_{m}, \quad a_{m}, \quad a_{0} .
$$

By a theorem* of Dickson's, any symmetric function of these three expressions is a formal invariant, modulo 2 , all such being, of course, rational in the three elementary symmetric functions

$$
\begin{gathered}
K=a_{1}+a_{2}+\cdots+a_{m-1}, \quad I=a_{0} a_{m}+\left(a_{0}+a_{m}\right)\left(a_{0}+a_{m}+K\right), \\
k=a_{0}\left(a_{0}+K+a_{m}\right) a_{m} .
\end{gathered}
$$

In the same way if

$$
C_{M}=A_{0} x_{1}^{M}+A_{1} x_{1}^{M-1} x_{2}+\cdots
$$

is any formal covariant of $f_{m}$, modulo 2 , any symmetric function of

$$
A_{0}+\cdots+A_{M}, \quad A_{0}, \quad A_{M}
$$

is an invariant of the original form $f_{m}$. Thus the middle coefficient of any quadratic covariant of $f_{m}$ is an invariant, the sum of the two middle coefficients of any cubic covariant is an invariant, and so on.

By means of the transformations on the $a$ 's induced by $x_{1}=x_{1}^{\prime}+t x_{2}^{\prime}$, $x_{2}=x_{2}^{\prime}$, viz.

(5) $a_{r}^{\prime}=\left[\left(\begin{array}{c}m \\ r\end{array}\right) a_{0}+\left(\begin{array}{c}m-1 \\ r-1\end{array}\right) a_{1}+\cdots+\left(\begin{array}{c}m-r+1 \\ 1\end{array}\right) a_{r-1}\right] t+a_{r}$

it is readily shown that

$$
(r=0, \cdots, m) \text {, }
$$

$$
K_{2}=a_{0} x_{1}^{2}+K x_{1} x_{2}+a_{m} x_{2}^{2}, \quad K_{1}=\left(a_{0}+K\right) x_{1}+\left(K+a_{m}\right) x_{2},
$$

are formal covariants modulo 2 .

LEMma. For any odd order $m>1(p=2)$ the form $f_{m}$ has a cubic covariant $K_{m 3}$ with leading coefficient $a_{0}$, which is such that

$$
K_{m 3} \equiv K_{2} \quad(\bmod 2)
$$

for integral values of $x_{1}, x_{2}$.

* These Trans a c tion s, vol. 15 (1914), p. 497. 
The covariants $K_{m 3}$ for the first twelve odd orders $m$ are

$$
\begin{aligned}
K_{33} & =a_{0} x_{1}^{3}+a_{1} x_{1}^{2} x_{2}+a_{2} x_{1} x_{2}^{2}+a_{3} x_{2}^{3}, \\
K_{53} & =a_{0} x_{1}^{3}+\left(a_{1}+a_{2}\right) x_{1}^{2} x_{2}+\left(a_{3}+a_{4}\right) x_{1} x_{2}^{2}+a_{5} x_{2}^{3}, \\
K_{73} & =a_{0} x_{1}^{3}+\left(a_{1}+a_{2}+a_{4}\right) x_{1}^{2} x_{2}+\left(a_{3}+a_{5}+a_{6}\right) x_{1} x_{2}^{2}+a_{7} x_{2}^{3}, \\
K_{93}= & a_{0} x_{1}^{3}+\left(a_{1}+a_{2}+a_{3}+a_{4}\right) x_{1}^{2} x_{2}+\left(a_{5}+a_{6}+a_{7}+a_{8}\right) x_{1} x_{2}^{2}+a_{9} x_{2}^{3}, \\
K_{113}= & a_{0} x_{1}^{3}+\left(a_{1}+a_{2}+a_{4}+a_{5}+a_{8}\right) x_{1}^{2} x_{2} \\
& \quad+\left(a_{3}+a_{6}+a_{7}+a_{9}+a_{10}\right) x_{1} x_{2}^{2}+a_{11} x_{2}^{3} .
\end{aligned}
$$

The general form to be proved is

where

$$
K_{m 3}=a_{0} x_{1}^{3}+I_{1} x_{1}^{2} x_{2}+I_{2} x_{1} x_{2}^{2}+a_{m} x_{2}^{3},
$$

$$
\begin{array}{cl}
I_{1}=a_{i_{1}}+\cdots+a_{i_{\mathrm{e}}}, \quad & I_{2}=a_{j_{1}}+\cdots+a_{j_{\mathrm{\iota}}}, \quad s=\frac{1}{2}(m-1), \\
& I_{1}+I_{2}=K .
\end{array}
$$

Assuming the increments of $I_{1}, I_{2}$ under (5) to be $I_{1}^{\prime} t, I_{2}^{\prime} t$ we readily show that

$$
I_{1}^{\prime} \equiv I_{2}^{\prime} \equiv a_{0} \quad(\bmod 2)
$$

Hence, letting $I_{1}=\alpha_{1} a_{1}+\cdots+\alpha_{m-1} a_{m-1}\left(\alpha_{j}=0\right.$ or 1$)$, we have from $\sum_{r=1}^{r=m-1} \alpha_{r} a_{r}^{\prime}$, with (5), the conclusion that the selection of the set $a_{i_{1}}, a_{i_{2}}$, $\cdots, a_{i}$ is accomplished by selecting a set $\sigma_{1}$ of columns from the following Pascal triangle (6), the set to have the properties $(a),(b)$ below:

$$
\begin{aligned}
& \left(\begin{array}{c}
m-1 \\
1
\end{array}\right)\left(\begin{array}{c}
m-1 \\
2
\end{array}\right) \cdots\left(\begin{array}{l}
m-1 \\
m-3
\end{array}\right)\left(\begin{array}{l}
m-1 \\
m-2
\end{array}\right), \\
& \left(\begin{array}{c}
m \\
1
\end{array}\right) \quad\left(\begin{array}{c}
m \\
2
\end{array}\right) \quad\left(\begin{array}{c}
m \\
3
\end{array}\right) \quad \cdots\left(\begin{array}{c}
m \\
m-2
\end{array}\right)\left(\begin{array}{c}
m \\
m-1
\end{array}\right) \text {, } \\
& \begin{array}{llllll}
a_{1} & a_{2} & a_{3} & \cdots & a_{m-2} & a_{m-1} .
\end{array}
\end{aligned}
$$

(a) The sum of the numbers of the first row of the selected set $\sigma_{1}$ is odd.

(b) The sum for all of the other rows of $\sigma_{1}$ is even.

Grant for the moment that such a set can be selected. If its columns be deleted from (6) the set $\sigma_{2}$ of columns remaining in (6) will also have the properties $(a),(b)$ since Pascal's triangle is symmetrical with respect to the median drawn from ( $\left(\begin{array}{l}2 \\ 1\end{array}\right)$. This second set $\sigma_{2}$ gives $a_{j_{1}}, a_{j_{2}}, \cdots, a_{j_{\text {. }}}$, i. e., $I_{2}$. We now construct the triangle $A B C$ made up of the residues modulo 2 of 
Pascal's triangle, placing $\left(\begin{array}{l}2 \\ 1\end{array}\right)$ at the upper vertex, with the aforesaid median drawn vertically. We note particularly the symmetrical properties, and in particular the regular recurrence of the inverted triangles of zeros, of increasing dimensions, having the median as a line of symmetry. Consider any element $e$ of any horizontal row, to the left of the median. We call the column parallel to $A B$ and containing $e$ the column of $e$. The element $e^{\prime}$ in the same row as $e$ but occupying the complementary position to the right of the median is called the complementary element of $e$. The coliumn parallel to $A B$ containing $e^{\prime}$ is the complementary row of $e$, and the column containing $e$ and parallel to $B C$ is the complementary column of $e$. We have $e^{\prime}=e$, and the complementary row and complementary column of $e$ identical.

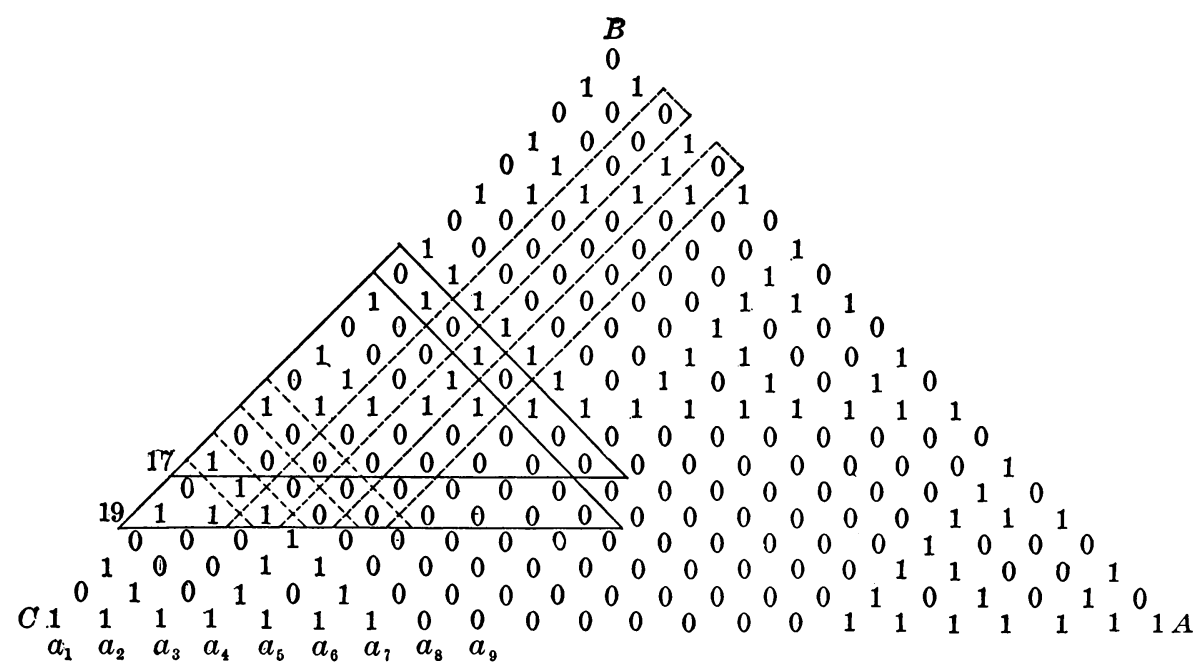

Suppose we wish to select $I_{1}$, i. e., the set $\sigma_{1}$, for $m=19$. We take the left-hand half-row number 18 from $B$ as hypotenuse and draw the triangle enclosing the columns of these elements. The elements on the hypotenuse correspond to set

$$
T_{1}: a_{1}, a_{2}, a_{3}, a_{4}, a_{5}, a_{6}, a_{7}, a_{8}, a_{9} .
$$

If the rows enclosed within this triangle satisfy conditions $(a),(b)$ (as is the case for $m=17$ ) then $\sigma_{1}$ would be the set $T_{1}$ and $I_{1}$ equal to the sum of the $\frac{1}{2}(m-1) a$ 's in their natural order beginning with $a_{1}$. The sums of the rows above the first in the triangle are however $1,1,0,1,0,1,0,1(\bmod 2)$ respectively, instead of $0,0,0,0,0,0,0,0(\bmod 2)$ required by condition (b). To secure a triangle (augmented) which does satisfy $(b)$ we replace some of the elements on the hypotenuse by their complementary elements, in this case $e_{3}, e_{5}$ (corresponding to $\left.a_{3}, a_{5}\right)$ by $e_{3}^{\prime}, e_{5}^{\prime}$ (corresponding to $a_{16}, a_{14}$ ). 
The columns of $e_{3}, e_{5}$ are thus replaced by their complementary columns by rotating the latter counter-clockwise around $e_{3}, e_{5}$. The augmented triangle now satisfies conditions $(a),(b)$ and hence

$$
I_{1}=a_{1}+a_{2}+a_{16}+a_{4}+a_{14}+a_{6}+a_{7}+a_{8}+a_{9} .
$$

Now the orders $m$ for which the triangles do not need to be augmented are those giving the first row below the base of each central triangle of zeros. These values of $m$ are

$$
3,5,9,17,33, \cdots, 2^{n}+1, \cdots
$$

and $2^{n}-1$ is the number of the row, counting downward from $B$, in which the base of the $n$th central triangle of zeros is found. For $m=2^{n}+1, I_{1}$ equals the sum of the first $2^{n-1} a$ 's in their natural order, $I_{1}=a_{1}+a_{2}+\cdots$. Between any two consecutive cases of (7) there is a cycle of augmented triangles such that the whole configuration from $B$ downward forms a sequence of recurring cycles of figures proceeding according to the laws of symmetry of the triangle $A B C$. Thus the set $\sigma_{1}$ can always be selected and the lemma is true.

We now observe that any covariant $C_{M}$ of $f_{m}$ of odd order $M$ has corresponding to it a quadratic and a cubic covariant of $f_{m}$, constructed as covariants of $C_{M}$, on the models of $K_{2}, K_{m 3}$ respectively. (Cf. $\S 6$.)

\section{The Finiteness of the FORMal CONCOMitants For the ORDER $m$ AND MODULUS 2}

Theorem. The general quantic $f_{m}, m>3$, is reducible, modulo 2 , in terms of $Q, L$, and its own covariants of the first degree and orders $1,2,3$.

Let $m$ be even, $m=2 k$. Then $f_{m}-Q^{k-1} K_{2}$ has the factor $x_{2}$. Hence it has the factor $L$, since the real points $(\bmod 2)$ form a conjugate set under the group $G$. Therefore

$$
f_{m} \equiv Q^{k-1} K_{2}+L C \quad(\bmod 2),
$$

where $C$ is a first degree covariant of $f_{m}$ of odd order $2 k-3$. Next let $m$ be odd, $m=2 h+3>3$. Then

$$
f_{m} \equiv Q^{h} K_{m 3}+L C^{\prime} \quad(\bmod 2),
$$

where $C^{\prime}$ is of even order $2 h$.

The forms $C, C^{\prime}$ are, in turn, reducible in terms of their own covariants of orders 2,3 with $L$ and $Q$, and, as a covariant of $C$ is a covariant of $f_{m}$, we are led to a recurring process by which $f_{m}$ is in all cases expressed in terms of its covariants of orders $1,2,3$, and $L$ and $Q$, which was to be proved.

Any concomitant of a polynomial in a set of concomitants is a function of 
concomitants of the forms in the set. Hence if the systems for the orders $1,2,3$, and the simultaneous systems modulo 2 for these three orders are finite, the system for $f_{m}$ is likewise finite (see $\S 6$ ).

\section{Systems OF THE FIRST DEGREE}

While illustrating formulas (8), (9), we now derive fundamental systems of first degree concomitants for the quartic and the quintic forms.

An independent set of linear seminvariants of $f_{4}$ is $a_{0}, a_{1}, a_{2}+a_{3}$. The only linear invariant is $C_{020}=a_{1}+a_{2}+a_{3}(=K, m=4(\S 3))$. Multiplication of $K_{1}$ of $\left(5_{1}\right)$ and $C_{101}$ of $\S 1$ by powers of $Q$ gives covariants of any odd order led by $a_{1}, a_{0}+K$, and, by subtracting such covariants, covariants led by $a_{0}+a_{2}+a_{3}$.

Lemma. There exists no covariant of odd order led by $K$.

Assume such a covariant in the form $C=K x_{1}^{2 h+1}+I x_{1}^{2 h} x_{2}+\cdots$. Then from (5), $m=4$,

$$
K\left(x_{1}^{2 h+1}+x_{1}^{2 h} x_{2} t+\cdots\right)+I^{\prime}\left(x_{1}^{2 h} x_{2}+\cdots\right) \equiv C \quad(\bmod 2),
$$

where $I$ becomes $I^{\prime}$ under (5). Hence, writing $I^{\prime} \equiv I+t I_{1}$,

$$
K \equiv I_{1} \quad(\bmod 2)
$$

Now if $I$ contains $a_{4}$, the increment $I_{1}$ contains $a_{0}$, whereas $K$ does not. Hence $I$ does not contain $a_{4}$. But the increment to a linear function of $a_{0}, a_{1}, a_{2}, a_{3}$ contains $a_{1}$ only, whereas $K$ contains $a_{2}$. Hence $K \neq I_{1}$, a contradiction.

It now follows that the general form of a covariant of odd order is

Thus

$$
C=\left[\lambda a_{1}+\mu\left(a_{0}+a_{2}+a_{3}\right)\right] x_{1}^{2 k+1}+\cdots \text {. }
$$

$$
C \equiv(\lambda+\mu) Q^{k} C_{101}+\mu Q^{k} K_{1}+L \Gamma \quad(\bmod 2),
$$

where $\Gamma$ is of even order $2 k-2$.

Any covariant of even order may be written

$$
C_{1}=\left[\lambda a_{0}+\mu a_{1}+\nu\left(a_{2}+a_{3}\right)\right] x_{1}^{2 h}+\cdots,
$$

and we have, using $K_{2}(m=4)$ of $\S 3$, and $C_{012}$ of $\S 1$,

$$
C_{1} \equiv Q^{h-1}\left[\lambda K_{2}+(\mu+\nu) C_{012}\right]+\nu K Q^{h}+L \Gamma_{1} \quad(\bmod 2) ;
$$

$\Gamma_{1}$ being a first degree covariant of odd order $2 h-3$. Hence the fundamental system sought is

$$
K, K_{1}, K_{2}, C_{101}, C_{012}, L, Q \text {. }
$$

The form $f_{4}$ itself is reducible as follows [cf. (8)]:

$$
f_{4} \equiv Q K_{2}+L\left(K_{1}+C_{101}\right) \quad(\bmod 2) .
$$


Fimploying $K_{1}, K_{2}, K, K_{m 3}(m=5)$ of $\S 3$ and $C_{021}, C_{102}$ of $\S 1$, we find for the required fundamental system of the quintic,

$$
K, K_{1}, K_{2}, K_{53}, C_{021}, C_{102}, L, Q \text {. }
$$

The reduction of the form $f_{5}$ itself is given by

$$
f_{5} \equiv Q K_{53}+L\left(K_{2}+C_{102}\right) \quad(\bmod 2) \quad \text { [cf. (9)]. }
$$

6. ON THE COMPLETE SYSTEM OF THE CUBIC, MODUlo 2

The development of the asyzygetic theory of the concomitants of a form often precedes the derivation of its complete system. In this section we show that every covariant of order $>3$ of a binary cubic, modulo 2 , is quasireducible (i. e., reducible on multiplication by $K^{s}(s \geqq 0)$ ) in terms of a set of fourteen concomitants, nine covariants and five invariants.

It is known that the fundamental system of seminvariants* of $f_{3}$ modulo 2 is

$$
\begin{gathered}
a_{0}, \quad K=a_{1}+a_{2}, \quad \delta_{00}=\left(a_{0}+K+a_{3}\right) a_{3}, \\
\Delta=a_{0} a_{3}+a_{1} a_{2}, \quad \beta=a_{1}^{2}+a_{0} a_{1},
\end{gathered}
$$

and also that $f_{3}$ has the invariants

$$
\begin{gathered}
K, \quad \Delta, \quad I=a_{0}^{2}+a_{0} K+\delta_{00}, \quad k=a_{0} \delta_{00}, \\
V=\beta\left(\beta+K^{2}+a_{0} K\right)\left(\Delta+\delta_{00}\right) .
\end{gathered}
$$

In two previous papers (quoted above) I have shown that $f_{3}$ has the covariants $\dagger$

$$
\begin{aligned}
H & =s x_{1}^{2}+\Delta x_{1} x_{2}+\left(a_{1} a_{3}+a_{2}^{2}\right) x_{2}^{2}, \\
G_{1} & =(\Delta+s) x_{1}+\left(\Delta+a_{1} a_{3}+a_{2}^{2}\right) x_{2} \\
P & =a_{0}^{2} x_{1}^{3}+a_{1}^{2} x_{1}^{2} x_{2}+a_{2}^{2} x_{1} x_{2}^{2}+a_{3}^{2} x_{2}^{3}, \quad\left(s=a_{0} a_{2}+a_{1}^{2}\right), \\
K_{1} & =\left(a_{0}+K\right) x_{1}+\left(K+a_{3}\right) x_{2} \\
K_{2} & =a_{0} x_{1}^{2}+K x_{1} x_{2}+a_{3} x_{2}^{2} \\
C_{1} & =\left(a_{0}^{2}+K^{2}\right) x_{1}+\left(K^{2}+a_{3}^{2}\right) x_{2} \\
C_{2} & =a_{0}^{2} x_{1}^{2}+K^{2} x_{1} x_{2}+a_{3}^{2} x_{2}^{2} .
\end{aligned}
$$

There is, therefore, a covariant led by $\beta$, viz.,

$$
t=K K_{2}+H=\beta x_{i}^{2}+\left(\Delta+K^{2}\right) x_{1} x_{2}+\left(a_{2} a_{3}+a_{2}^{2}\right) x_{2}^{2} .
$$

* Dickson, Madison Colloquium Lectures, p. 53.

†Cf. American Journal of Mathematics, loc. cit., p. 78. The forms $C_{4}^{(1)}, C_{2}^{(3)}$ in Table $\mathrm{I}$ are reducible as follows:

$$
C_{4}^{(1)} \equiv L K_{1}+Q K_{2}, \quad C_{2}^{(3)} \equiv K t+\left(\Delta+K^{2}\right) K_{2}(\bmod 2) .
$$


None of the invariants $\Delta, g$ (below), $I$ can be leading coefficients of covariants of odd order, but there exists a cubic covariant led by the invariant $K$, viz., $G=Q K_{1}+f_{3}=K x_{1}^{3}+\left(a_{0}+a_{1}+a_{3}\right) x_{1}^{2} x_{2}+\left(a_{0}+a_{2}+a_{3}\right) x_{1} x_{2}^{2}+K x_{2}^{3}$.

Taking the elementary symmetric function of second degree in the coefficients of $t$, as explained in $\S 3(4)$, we have the fourth degree invariant of $f_{3}$,

$$
\begin{aligned}
g & =\beta^{2}+\beta\left(\Delta+K^{2}\right)+\beta \beta_{1}+\beta_{1}\left(\Delta+K^{2}\right)+\beta_{1}^{2} \quad\left(\beta_{1}=a_{2} a_{3}+a_{2}^{2}\right), \\
& \equiv \beta^{2}+\beta\left(\Delta+K^{2}\right)+\left(\Delta+\delta_{00}\right)\left(\beta+a_{0} K+K^{2}\right) \quad(\bmod 2) .
\end{aligned}
$$

Now from (13), (15) we have

$$
\begin{aligned}
a_{0}^{3}+ & a_{0}^{2} K+a_{0} I+k \equiv 0 \\
\beta^{2}+\beta\left(a_{0}^{2}+a_{0} K+I\right. & \left.+K^{2}\right) \\
& +\left(a_{0}^{2}+a_{0} K+I+\Delta\right)\left(a_{0} K+K^{2}\right)+g \equiv 0 .
\end{aligned}
$$

Any seminvariant $\phi$ of $f_{3}$, being a polynomial in the seminvariants (12), is a polynomial in $a_{0}, K, \beta, \Delta, I$;

$$
\phi=\phi\left(a_{0}, \beta, K, \Delta, I\right) .
$$

We now use congruences (16) as reducing moduli, whereupon we are able to reduce all exponents* of $\beta$ (in $\phi$ ) below 2 and all exponents of $a_{0}$ below 3 . Thus any seminvariant can be reduced to the form

$$
\phi=J_{0}+J_{1} a_{0}+J_{2} a_{0}^{2}+\left(\Gamma_{0}+\Gamma_{1} a_{0}+\Gamma_{2} a_{0}^{2}\right) \beta,
$$

wherein $J_{i}, \Gamma_{i}(i=0,1,2)$ are invariants (expressed in terms of $K, \Delta, k$, $I, g)$ some of which might be zero. Let $C_{M}$ be any covariant of $f_{3}$ of even order $M=2 h$, led by $\phi ; C_{M}=\phi x_{1}^{2 h}+\cdots$. Then,

$$
\begin{aligned}
C_{M} \equiv Q^{h} J_{0}+Q^{h-1} & \left(K_{2} J_{1}+C_{2} J_{2}\right) \\
& +Q^{h-1} t \Gamma_{0}+Q^{h-2}\left(K_{2} \Gamma_{1}+C_{2} \Gamma_{2}\right) t+L C \quad(\bmod 2),
\end{aligned}
$$

where $C$ is a covariant of odd order $2 h-3$. Thus every covariant of even order $>3$ is reducible in terms of our covariants and invariants.

Proceeding to covariants of odd order; there is a covariant with seminvariant leader $B=\beta+\Delta$, viz.,

$$
\begin{aligned}
& l=B x_{1}^{3}+\left(a_{0} a_{2}+a_{1} a_{2}+a_{1} a_{3}+a_{2}^{2}\right) x_{1}^{2} x_{2} \\
& \quad+\left(a_{0} a_{2}+a_{1} a_{2}+a_{1} a_{3}+a_{1}^{2}\right) x_{1} x_{2}^{2}+\left(\Delta+a_{2} a_{3}+a_{2}^{2}\right) x_{2}^{3} \\
& \\
& \equiv Q G_{1}+K f_{3} .
\end{aligned}
$$

* Cf. L. J. Reed, "Some fundamental systems of formal modular invariants and covariants," Dissertation, University of Pennsylvania, 1915, § 3. 
Replacing $\beta$ by $B+\Delta$ in (16) and in $\phi$ we can reduce any seminvariant to the form

$$
\phi=R_{0}+R_{1} a_{0}+R_{2} a_{0}^{2}+\left(S_{0}+S_{1} a_{0}+S_{2} a_{0}^{2}\right) B,
$$

where $R_{i}, S_{i}(i=0,1,2)$ are polynomials in the five invariants $K, \Delta, k$, $I, g$. Let $C_{N}$ be a covariant of $f_{3}$ of odd order $N=2 h+3>3$, led by $\phi$; $C_{N}=\phi x_{1}^{N}+\cdots$. Then

$K C_{N} \equiv Q^{h} R_{0} G+K R_{1} Q^{h} f_{3}+K R_{2} Q^{h} P$

$$
+K\left(l S_{0} Q^{h}+l S_{1} K_{2} Q^{h-1}+l S_{2} C_{2} Q^{h-1}\right)+L C^{\prime}
$$

We have now proved the following:*

THEOREM: Every formal covariant modulo 2 of order $>3$ of the binary cubic $f_{3}$ is quasi-reducible, upon multiplication by $K^{s}(s \geqq 0)$, in terms of the fourteen concomitants listed below:

$$
K, \Delta, k, I, g, f_{3}, K_{2}, C_{2}, K_{1}, G_{1}, P, t, L, Q .
$$

* On the subject of quasi-reducibility in ternariant theory, cf. Forsyth, Am e ri c a n Journal of Mathematics, vol. 12 (1890).

University of Pennsyluania 